\title{
AR/VR.nrw - Augmented und Virtual Reality in der Hochschullehre
}

\author{
Sinja Müser (D) Christian Dominic Fehling
}

Eingegangen: 16. Juli 2021 / Angenommen: 17. November 2021 / Online publiziert: 9. Dezember 2021

(C) Der/die Autor(en) 2021

Zusammenfassung Immer mehr Hochschulen suchen nach Lösungen Augmented und Virtual Reality (AR/VR) stärker in Lehre und Forschung einzubinden. Es wurde bereits gezeigt, dass für manche Sachverhalte immersive Technologien verglichen mit traditionellen Lernmethoden besser geeignet sind; speziell um komplexe Sachverhalte zu erleben und zu lernen. Abseits solcher Projekte finden sich jedoch kaum Fälle einer breiten Anwendung in der gängigen Hochschullehre. Mit diesem Beitrag wird eine Möglichkeit vorgestellt, Augmented und Virtual Reality stärker und allgemein verständlicher in die Lehre und Forschung der Hochschulen zu integrieren und eine verbesserte Kollaboration zwischen den beteiligten Akteuren und Stakeholdern zu ermöglichen. Vor dem Hintergrund lehr-lernpsychologischer und mediendidaktischer Theorien sowie auf Basis von Wirksamkeitsstudien wird daher mit Figments.nrw eine offene und freie Lösung entwickelt, die sowohl eine Autorenumgebung zur einfachen Erstellung von Augmented- und Virtual-Reality-Inhalten als auch Instrumente zur Durchführung von Lehr-/Lernaktivitäten umfasst. Hierbei werden Erkenntnisse und Erfahrungen aus der Nutzung sowie Untersuchung bisheriger Lösungen, wie notwendige informationstechnische Expertise, RessourcenIntensität sowie monolithische und proprietäre Software, berücksichtigt.

Schlüsselwörter AR/VR · Autorenwerkzeuge $\cdot$ Open Source $\cdot$ Immersives Lernen · Instruktionsdesign

\footnotetext{
Sinja Müser $(\square)$

Hochschule Hamm-Lippstadt, Marker Allee 76-78, 59063 Hamm, Deutschland

E-Mail: sinja.mueser@hshl.de

Christian Dominic Fehling

Bergische Universität Wuppertal, Rainer-Gruenter-Straße 21, 42119 Wuppertal, Deutschland

E-Mail: fehling@uni-wuppertal.de
} 


\title{
AR/VR.nrw-Augmented and Virtual Reality in Higher Education
}

\begin{abstract}
More and more universities are looking for solutions to integrate augmented and virtual reality (AR/VR) into teaching and research. It has already been shown that for some subjects immersive technologies are better suited compared to traditional learning methods, especially for experiencing and learning complex subjects. However, apart from such projects, there are hardly any cases of a broad application in common university teaching. This paper presents a possibility of integrating augmented and virtual reality more strongly and in a more generally understandable way into teaching and research of universities and enabling improved collaboration between the involved actors and stakeholders. Against the background of theories of instructional psychology and media didactics as well as on the basis of effectiveness studies, Figments.nrw is therefore being developed as an open and free solution that includes both an authoring environment for the simple creation of augmented and virtual reality content as well as tools for the implementation of teaching/learning activities. Here, insights and experiences from the handling and investigation of previous solutions, such as necessary information technology expertise, resource intensity, and monolithic and proprietary software, are considered.
\end{abstract}

Keywords AR/VR · Authoring Tools · Open Source · Immersive Learning · Instructional Design

\section{Lehren und Lernen mit Augmented und Virtual Reality}

In den letzten 50 Jahren haben digitale Medien zunehmend Einzug in den Bildungsbereich gehalten und bieten Lernenden durch interaktive Simulationen, Lernspiele und Ähnliches umfangreiche Lernmöglichkeiten. Ergänzend sind mit Augmented und Virtual Reality (AR/VR) in den letzten Jahren immersive Medien hinzugekommen, denen ein hohes Potenzial zur weiteren Verbesserung des Lehrens und Lernens und zur Realisierung neuartiger Lehr- und Lernszenarien zugesprochen wird (Hellriegel und Čubela 2018; Köhler et al. 2013; Zender et al. 2018).

Eine einheitliche Definition von AR und VR sucht man bislang vergeblich. Dies erscheint vor allem vor dem Hintergrund schwierig, da diese Begriffe sowohl aus technologiezentrierten sowie methodologischen Perspektiven betrachtet werden können als auch unterschiedliche Zielvorstellungen (Forschung, Unterhaltung, Wirtschaftlichkeit) verfolgt werden können. In Anlehnung an Blade und Padgett (2015) und Dörner et al. (2013) lassen sich AR und VR jedoch wesentlich und in Kürze wie folgt beschreiben und unterscheiden: AR (Augmented Reality, d.h. ,erweiterte Realität") bezeichnet eine computerunterstützte Wahrnehmung bzw. Darstellung, in welcher die reale Umgebung von digital erstellten Inhalten überlagert wird. In AR bleibt die reale Welt also erhalten und wird in Echtzeit, interaktiv und positionsgenau (Azuma et al. 2001) um virtuelle Elemente ergänzt. Im Vergleich dazu bezeichnet VR (Virtual Reality, d.h. ,virtuelle Realität“) eine computergenerierte Wirklichkeit, die Anwenderinnen und Anwender vollständig umgibt, sie können in diese virtuelle Umgebung abtauchen. 
In Anbetracht der steigenden Bedeutung von AR und VR bei der Gestaltung von Lehr- und Lernprozessen (Goertz et al. 2021), ist der aktuelle Stand der Entwicklung, die Einbindung in die (Hochschul-)Lehre sowie die Evaluation der Wirksamkeit jedoch noch nicht zufriedenstellend erforscht (Niedermeier und Müller-Kreiner 2019). Gründe hierfür sind sowohl technische Herausforderungen, wie ein Mangel an Kompatibilität zwischen Systemen oder auch der Umstand, dass die meisten Entwicklungen nicht Open-Source zur Verfügung stehen. Auch individuelle Faktoren wirken sich negativ aus, da bisherige AR/VR-Systeme meist tiefgehende interdisziplinäre Expertise in den Bereichen Softwareentwicklung, 3D-Modellierung, Kompetenzen im Medienumgang und der Anwendung digitaler Medien in Unterrichtsszenarien voraussetzen und viele Kapazitäten binden. Neben der aktuell nicht zufriedenstellenden Nutzung beziehungsweise Nutzbarkeit von AR/VR-Systemen in diesem Zusammenhang, liegen außerdem kaum didaktische Konzepte zur Realisierung solcher Lernumgebungen vor (Hochberg et al. 2017; Zender et al. 2018), obwohl deren Einsatz nicht lediglich technologiegetrieben sein sollte, sondern einen Mehrwert gegenüber anderen Formaten bieten und ein konkretes Bildungsinteresse verfolgen sollte (Kerres 2018).

Vor diesem Hintergrund eröffnet das Projekt AR/VR.nrw (siehe Infokasten) zwei Perspektiven für AR/VR-Lösungen, die sowohl konzeptionell als auch mit Blick auf erste Zwischenergebnisse im Zentrum des Beitrags stehen.

1. Der Einsatz von Augmented und Virtual Reality in der Hochschulbildung muss didaktisch und technisch fundiert sein. Hierzu werden Grundlagen für ein technisch-didaktisches Konzept erarbeitet, das durch zusätzliche Trainingsmaterialien (Screencasts, Handbücher, E-Learnings) und die Einbindung in akademische Weiterbildungsstrukturen flankiert wird. Hierbei werden Akteure aus unterschiedlichsten Bereichen der Hochschulbildung einbezogen, deren Feedback in die Weiterentwicklung und Verbesserung der Lösung einfließt (Kap. 2 und 4).

2. Es wird eine Lösung entwickelt, erprobt und zur Verfügung gestellt, die Lehrende mit geringer IT-Expertise nutzen und mit eigenen, unterschiedlichsten Lehrinhalten füllen können. Diese wird offen und modular entwickelt, sodass Forscherinnen und Forscher sowie Entwicklerinnen und Entwickler diese für neue Forschungsund Lehrzwecke weiterentwickeln können. Der dabei entstehende Quellcode wird - auch zur kollaborativen Weiterentwicklung nach Ende der Förderphase des Projektes - soweit wie möglich schon während der Entwicklungsphase veröffentlicht (Kap. 3).

AR/VR.nrw Im Projekt AR/VR.nrw werden die Technologien Augmented und Virtual Reality stärker in Lehre und Forschung der Hochschulen integriert und die Zusammenarbeit in diesem Bereich verbessert. Hierzu wird mit Figments.nrw eine offene und freie Lernsoftware und Autorenumgebung entwickelt und zur Verfügung gestellt. Ergänzend werden exemplarische Lernszenarien erarbeitet und Services für Hochschulen aufgebaut, die Lehrende bei der Nutzung und Entwicklung von Augmented und Virtual Reality in der Hochschule unterstützen. 
- Konsortialführer: HS Bonn-Rhein-Sieg

- Konsortium: RWTH Aachen, Bergische Universität Wuppertal, HS Hamm-Lippstadt

- Laufzeit: 09/2020-08/2023

- Website: www.figments.nrw

\section{$2 \mathrm{AR} / \mathrm{VR}$ in der Hochschullehre}

Das steigende Interesse, vielversprechende Forschungsergebnisse sowie die zunehmende Finanzierbarkeit von Virtual Reality hat es Bildungseinrichtungen ermöglicht, die Technologie in ihre Lehre einzubinden. Darüber hinaus hat die steigende Leistung von Computern und zugehöriger Hardware zu einer Revolution in der grafischen Darstellung geführt. Obwohl AR/VR grundsätzlich nicht neu ist, haben die jüngsten Entwicklungen bei immersiven Technologien, die immer komplexere und realistische Simulationen, Visualisierungen und Interaktionen ermöglichen, AR/VR für Bildungszwecke zunehmend attraktiv gemacht. So beschäftigen sich zunehmend Hochschulen sowie Forschungsvorhaben mit der Nutzbarkeit und Relevanz von AR und VR für das Lehren und Lernen (Makransky und Petersen 2021; Goertz et al. 2021). Die Einführung von AR/VR in der akademischen Bildung bringt allerdings auch die Frage nach einer konzeptionellen Definition dessen mit sich, was eine Lernumgebung sinnvoll und erfolgreiches Lernen möglich macht. Bei der Konzeption neuer Lehr- und Lernmaterialien oder -angeboten müssen im Sinne des Instruktionsdesigns, unabhängig von der medialen Präsentation der Lerninhalte, einige didaktisch-konzeptionelle Faktoren berücksichtigt werden, um erfolgreiches Lernen zu ermöglichen. Besonders gilt dies für digitales Lehren und Lernen, da umfassendere Gestaltungs- und Interaktionsmöglichkeiten geboten werden und die Komplexität der Darbietung steigt. Vor allem um AR/VR-Lernumgebungen sinnvoll zu konstruieren und/oder in die Lehre zu integrieren, ist es von großer Bedeutung theoretisch und empirisch fundierte Gestaltungsgrundsätze zu verfolgen, um ein valides didaktischtechnisches Konzept zu erarbeiten.

\subsection{Lern- und Medientheorien}

Das Lernen und Verstehen von komplexen (naturwissenschaftlichen) Themen kann für viele Lernende eine große Herausforderung sein. Besonders für Lernende mit geringem Vorwissen, kann die Identifikation und Integration relevanter Aspekte des Lernmaterials, zur Konstruktion einer kohärenten mentalen Repräsentation, zu einer Überlastung des Arbeitsgedächtnisses führen. Aber auch steigende Komplexität sowie überflüssige Informationen können zu einer Steigerung der kognitiven Belastung führen und so das Lernen erschweren. Ein verbreiteter Vorschlag, um Kapazitäten im Arbeitsgedächtnis freizusetzen, ist die Verwendung von Lernmaterialien, die verschiedene inhaltliche Repräsentationen, wie beispielsweise Text und Illustrationen, sinnvoll kombinieren.

Dieser Vorschlag resultiert aus der Cognitive Theory of Multimedia Learning (CTML; Mayer 2014b), die das Lernen als generative kognitive Verarbeitung be- 


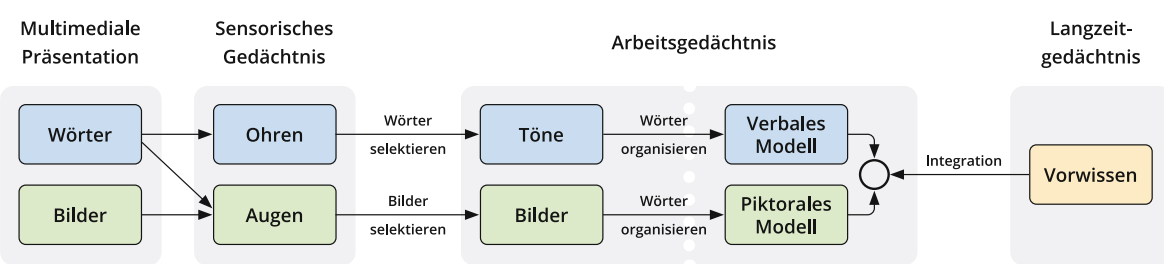

Abb. 1 Cognitive Theory of Multimedia Learning. (Mayer 2014b, S. 66; modifiziert, eigene Übersetzung)

schreibt (siehe Abb. 1) und auf den Annahmen basiert, dass (1) die Verarbeitung von visuellen und auditiven Informationen in zwei separaten Kanälen, dem piktoralen (z. B. Bilder oder Text) und dem verbalen (z.B. Klänge oder gesprochene Worte), erfolgt (Baddeley 2012; Clark und Paivio 1991), (2) die Menge an Informationen, die in jedem Kanal gleichzeitig verarbeitet werden kann, begrenzt ist (Baddeley 2012; Sweller 2010) und (3) sinnvolles Lernen eine angemessene kognitive Anstrengung während des Lernens sowie Aktivität des Lernenden beinhaltet (Mayer 2014a; Wittrock 1989).

In der Lehrpraxis bedeutet dies, dass Informationen vor allem dann gut erinnert werden können, wenn beispielsweise sowohl das Multimediaprinzip (die Kombination von verbalen und piktoralen Inhalten) als auch das Kontiguitätsprinzip (zeitlich und räumlich nahe Platzierung der Inhalte) bei der Unterrichtsgestaltung berücksichtigt wurden.

Bezüglich der Annahme 2 des CTML resultiert eine mögliche kognitive Überlastung aus der begrenzten Kapazität des Arbeitsgedächtnisses. Auf dieser Grundlage, das Arbeitsgedächtnis sei sowohl in der Kapazität (Verarbeitung von Informationen) als auch in der Zeit (Informationsspeicherung) beschränkt, basiert die Cognitive Load Theory (CLT; Paas und Sweller 2014; Sweller 2010). Der cognitive load, also die kognitive Belastung, ist die Menge und Art der Informationen, die das Gehirn zu einem bestimmten Zeitpunkt aufnehmen und speichern kann. Nach der CLT gibt es drei Kategorien kognitiver Belastung: intrinsic, extraneous und germane load (siehe Abb. 2).

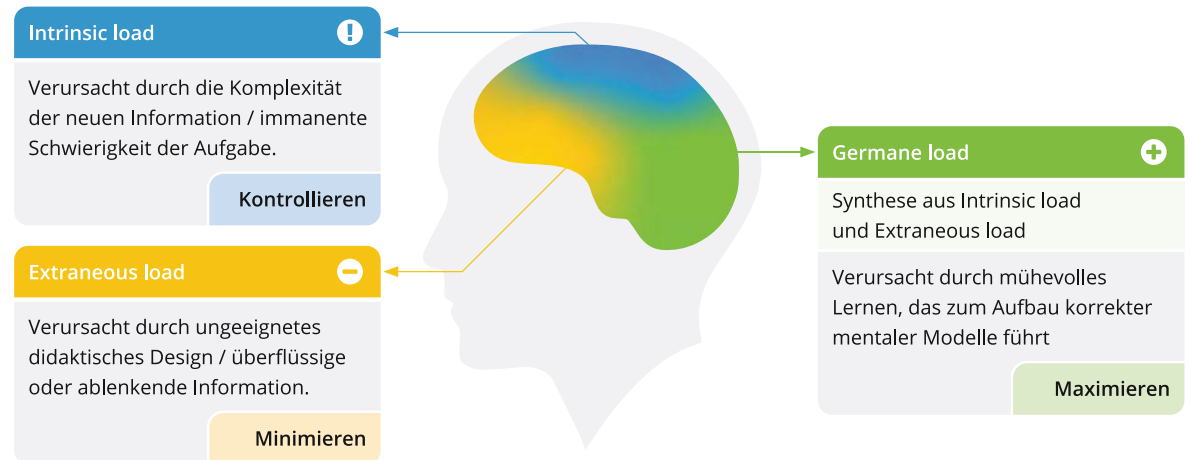

Abb. 2 Bildliche Darstellung der Cognitive Load Theory. (In Anlehnung an Paas und Sweller 2014) 
Intrinsic cognitive load (Sweller und Chandler 1994) ist die Belastung, die aus der immanenten Komplexität oder Schwierigkeit der Lernaufgabe resultiert; sie ist abhängig von der Interaktivität der Lernelemente sowie dem Vorwissen des Lernenden. Intrinsic cognitive load kann insofern nicht verändert werden, außer durch Modifizierung der Art der Aufgabe oder Vermehrung des Wissens (Paas und Sweller 2014).

Extraneous cognitive load ist die Belastung, die durch ungeeignetes didaktisches Design des Lehrmaterials hervorgerufen wird, wodurch Ressourcen für überflüssige Prozesse aufgebracht werden müssen. Das Hinzufügen von Material und Funktionen, wie z. B. visuelle Effekte in immersiver VR-Umgebung, kann somit zu höherem extraneous load führen. Diese kognitive Belastung kann und sollte durch eine sinnvolle didaktische Aufbereitung des Lehrmaterials reduziert werden.

Germane cognitive load bezeichnet die „effektive“ kognitive Belastung, da diese zeigt, dass der Lernende seine mentalen Ressourcen auf den tatsächlichen Lernprozess verwendet. Diese kognitive Belastung resultiert aus Lernendenaktivitäten (z. B. Erstellung von Mind-Maps o.ä.), die das Lernen erleichtern und zur Transferleistung beitragen, indem sie helfen, korrekte mentale Modelle aufzubauen (Paas et al. 2003).

Es ist davon auszugehen, dass je komplexer und vielfältiger die mediale Repräsentation der Lerninhalte ist, desto bedeutsamer werden das didaktische Design und die Reduktion der ineffektiven kognitiven Belastung. Hier können verschiedene Gestaltungsmethoden Anwendung finden (Lee et al. 2006; Mayer und Fiorella 2014; siehe Tab. 1).

Diese Prinzipien sind zunächst für multimediale Präsentationen von Lehrmaterial im Allgemeinen formuliert, lassen sich jedoch ebenso auf den Einsatz immersiver Medien übertragen. Auch hiermit werden Informationen textlich, bildlich, verbal oder anders präsentiert und müssen von den Lernenden aufgenommen und verarbeitet werden. Weitere immersiven Medien inhärente Faktoren, wie Interaktion, Intuition oder Kollaboration bedürfen jedoch weiterer Betrachtung.

Zusammenfassend profitieren Lernende somit von Lehrmaterialien, wenn sie aus den unterschiedlich dargebotenen Informationen (wie Text und Bild) ein kohärentes mentales Modell erstellen können, ohne sich dabei kognitiv zu überlasten (für einen

Tab. 1 Prinzipien zur Reduktion des extraneous load beim Lernen mit Multimedia

\begin{tabular}{|c|c|}
\hline Prinzip der ... & Lernende lernen besser ... \\
\hline Kohärenz & wenn fremdes Material eher ausgeschlossen als einbezogen wird \\
\hline Signalisierung & $\begin{array}{l}\text { wenn Hinweise hinzugefügt werden, die die Organisation des wesentlichen Inhal- } \\
\text { tes hervorheben }\end{array}$ \\
\hline $\begin{array}{l}\text { Redundanz } \\
\text { (vermeidung) }\end{array}$ & durch Grafik und Narration als durch Grafik, Narration und Bildschirmtext \\
\hline $\begin{array}{l}\text { räumliche Konti- } \\
\text { guität }\end{array}$ & $\begin{array}{l}\text { wenn korrespondierende Wörter und Bilder auf dem Bildschirm nahe beieinander } \\
\text { und nicht weit voneinander entfernt präsentiert werden }\end{array}$ \\
\hline $\begin{array}{l}\text { zeitliche Konti- } \\
\text { guität }\end{array}$ & $\begin{array}{l}\text { wenn entsprechende Animationen und Narrationen gleichzeitig und nicht nachein- } \\
\text { ander präsentiert werden }\end{array}$ \\
\hline Segmentierung & $\begin{array}{l}\text { wenn die Aufgabe in nutzergesteuerten Segmenten und nicht als durchgängige } \\
\text { Lerneinheit präsentiert wird }\end{array}$ \\
\hline
\end{tabular}


evidenzbasierten Überblick siehe Mayer 2014a). Hierzu bieten die CTML und die CLT Richtlinien für die didaktische Gestaltung von Lehr- bzw. Lernmaterial, die die spezifischen Merkmale der menschlichen kognitiven Architektur widerspiegeln. Folgt man diesen Richtlinien wird es den Lernenden ermöglicht, die Belastung des Arbeitsgedächtnisses zu regulieren und somit erfolgreich zu lernen. Neben zentralen Richtlinien wie diesen, die unabhängig von der Präsentation mittels herkömmlicher, digitaler oder immersiver Medien gelten, sollten bei der Nutzung immersiver Medien jedoch weiter Faktoren Berücksichtigung finden, welche das Lehren und Lernen beeinflussen. In Bezug auf das hier vorgestellte Vorhaben sind diese Faktoren von besonderer Bedeutung, weshalb diese nachfolgend im Detail betrachtet werden.

\subsection{Immersives Lernen}

Neben herkömmlichem (multimedialem und/oder digitalem) Lernmaterial, welches durchaus auch computerbasiert eingesetzt wird, geht die zu Bildungszwecken genutzte Technologie nun einen Schritt weiter. Vermehrt werden virtuelle Lernwelten genutzt, mithilfe derer Studierende sich mit Lerninhalten auseinandersetzen und überdies in diese eintauchen können.

Solche virtuellen Welten können über unterschiedliche Kanäle betreten werden: An konventionellen Desktop-Rechnern erschließen sie sich über die zweidimensionale Darstellung am Monitor, während mittels AR-respektive VR-Brille ein eintauchen in die Virtualität ermöglicht wird. Neben der Art der Interaktion, die in diesen Medien indirekt (über Maus und Tastatur) oder direkt und natürlich (über Hand-/ Body-Tracking) ausfallen kann, unterscheiden sich diese Zugänge vor allem im Grad der Immersion der erreicht wird. Während diese bei konventionellen DesktopAnwendungen relativ gering ausfällt, gelten VR-Anwendungen als hoch-immersiv (Buttussi und Chittaro 2018).

Nach Radianti et al. (2020) beschreibt Immersion „die Einbindung eines Anwenders in eine virtuelle Umgebung, bei der sein Zeitgefühl und die reale Welt oft abgekoppelt werden und stattdessen ein Gefühl des ,dort Seins ‘ in der Aufgabenumgebung entsteht" (S. 2; übersetzt).

Wie immersives Lernen bestmöglich gestaltet werden kann, beschreibt das Cognitive Affective Model of Immersive Learning (Makransky und Petersen 2021), indem es die bestehenden Forschungsergebnisse zur immersiven Bildung zusammenfasst, um den Prozess des Lernens in immersiver VR darzustellen.

\subsubsection{Cognitive Affective Model of Immersive Learning}

Auf der Basis früherer VR-basierter Forschung sowie von Motivations- und Lerntheorien, die für weniger immersive Medien aufgestellt wurden, entwickelten Makransky und Petersen (2021) das Cognitive Affective Model of Immersive Learning (CAMIL). Das CAMIL beschreibt, wie zwei Eigenschaften in immersiven VRUmgebung - Präsenzerleben, im Sinne eines Gefühls des , dort seins“ und Handlungsfähigkeit, im Sinne eines Gefühls Aktionen selbst zu erzeugen und zu steuern zu erfolgreichem Lernen führen. Weiter beschreibt das Modell, wie diese beiden Eigenschaften aus technologischen Merkmalen resultieren und wie das Lernen durch 


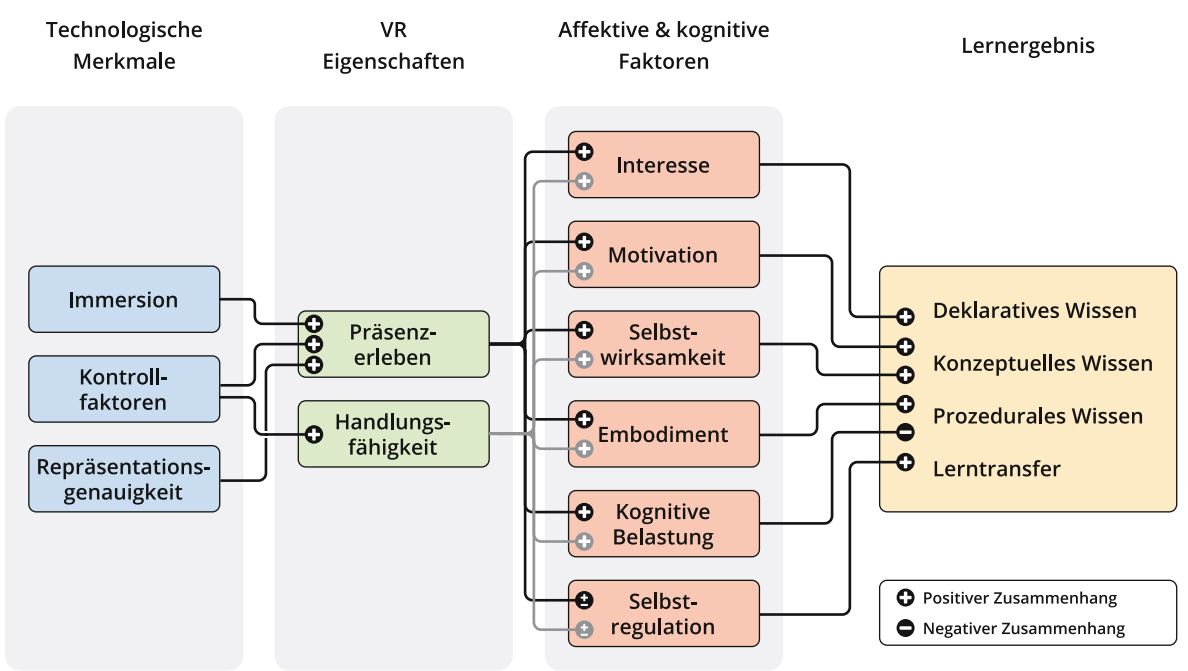

Abb. 3 Cognitive Affective Model of Immersive Learning. (Makransky und Petersen 2021, S. 7; eigene Übersetzung)

affektive und kognitive Prozesse beeinflusst wird. Abb. 3 veranschaulicht die im CAMIL enthaltenen Konstrukte sowie deren Zusammenhänge.

Das CAMIL basiert auf der theoretischen Perspektive, dass das Lehrmedium mit der Lehrmethode interagiert, was die Bedeutsamkeit einer planvollen Nutzung von Medien sowie deren sinnvolle didaktische Aufbereitung und Einbettung unterstreicht. Nach Makransky und Petersen (2021) sollte mindestens eine der beiden Eigenschaften, Präsenzerleben und/oder Handlungsfähigkeit, bei der Instruktion forciert werden, um die Interaktion von Medium und Methode anzustoßen. Die technologischen Merkmale der Immersion, Kontrollfaktoren (wie Grad, Direktheit und Art der Kontrolle) und Repräsentationsgenauigkeit (wie Realitätsnähe und Laufruhe der Darstellung, Konsistenz des Objektverhaltens), können dazu beitragen, das Präsenzerleben und die Handlungsfähigkeit von Lernenden im virtuellen Raum zu verbessern. Das Präsenzerleben und die Handlungsfähigkeit führen so über die affektiven und kognitiven Prozesse - Interesse, Motivation, Selbstwirksamkeit, Embodiment, kognitive Belastung und Selbstregulation - zum Lernergebnis, in Form von deklarativem, konzeptuellen und prozeduralem Wissen sowie Lerntransfer.

Mithilfe des CAMIL lassen sich für das didaktische Design von AR/VR-Lernmaterial folgende Implikationen ableiten (Makransky und Petersen 2021):

Lernmaterialien für den Einsatz in AR/VR sollten ...

- mit einem Fokus auf die didaktisch-methodischen Möglichkeiten von AR/VR, besonders Präsenzerleben und Handlungsfähigkeit, entwickelt werden,

- Faktoren zur Steigerung der Immersion, darunter Repräsentationsgenauigkeit und natürliche Interaktionen, hervorheben und besonders berücksichtigen, 
- für eine multimodale Ausgabe entworfen werden, bei der sowohl die Kollaboration von Lernenden über Mediengrenzen hinweg ermöglicht, als auch systeminhärente Medien- und Technologiepotenziale optimal ausschöpft werden,

- eine dem Anwendungsfall entsprechende realistische oder stilisierte Umgebungsgestaltung, Methoden zur Vermeidung von Simulationskrankheit und ein möglichst hohes $\mathrm{Maß}$ an (natürlichen) Interaktionen aufweisen.

Folgt man bei der didaktischen Konstruktion und Instruktion diesen Richtlinien, ist es möglich, realistische, erfahrungsbasierte Lernmöglichkeiten zu schaffen, die es den Lernenden ermöglichen Aufgaben auszuführen, die in der realen Welt unmöglich, unpraktisch oder zu teuer wären. Darüber hinaus gilt es ebenfalls, Lehr-, Lernsowie mediendidaktische Prinzipien zu berücksichtigen (wie CTML oder CLT), um so auch die affektiven und kognitiven Prozesse zu steuern.

\subsubsection{Wirksamkeit und Vorteile immersiver VR-Lernumgebungen}

Moreno und Mayer (2002) zeigten, dass die CTML nicht nur für nicht-immersive Medien, sondern auch für immersive Medien gilt. In einem Experiment zum Themenbereich Botanik wurde nachgewiesen, dass Studierende in einer virtuellen Lernumgebung besser Lernen, wenn neben grafischen Informationen gesprochene Erklärungen anstelle von textbasierten Einblendungen zum Einsatz kamen. Darüber hinaus zeigten sie, dass Studierende, welche die Lernumgebung per VR-Brille genutzt haben, ein signifikant stärkeres Präsenzgefühl erlebten, als Studierende, die die Lernumgebung zweidimensional am Desktop PC nutzen.

In einer Studie von Johnston et al. (2018) wurde der Wissenserwerb im Themenbereich Zellbiologie mittels immersiver VR-Umgebung untersucht. Es wurde nachgewiesen, dass diejenigen Studierenden, die zusätzlich zur entsprechenden Vorlesung eine virtuelle Zelle in VR erkunden konnten, signifikant besser in der Abschlussprüfung abschnitten, als Studierende, die ausschließlich an der Vorlesung teilgenommen haben.

Studien wie diese legen nahe, dass immersive VR-Lernumgebungen als Medium für akademisches Lernen ein potenzieller Ersatz/Zusatz für konventionelle Medien darstellen. Empirische Grundlagen finden sich hierzu bislang jedoch relativ selten. Aktuelle systematische Literaturreviews zeigen, dass die meisten Studien einen signifikanten Vorteil des Einsatzes von immersiver VR mittels HMD in der Lehre nahelegen, während eine kleinere Anzahl keine signifikanten Unterschiede im Leistungsniveau fand (Hamilton et al. 2021). Andere Reviews weisen zwar darauf hin, dass VR eine vielversprechende Möglichkeit für die Lehre zu sein scheint, deren Erforschung jedoch noch unzureichend ist. So weisen Radianti et al. (2020) darauf hin, dass in vielen Studien bei der Entwicklung von VR-Anwendungen kaum Lerntheorien berücksichtigt wurden, um das Lernen zu unterstützen und zu steuern. Außerdem konzentrierte sich die Evaluation im Bildungsbereich in erster Linie auf die Benutzerfreundlichkeit der VR-Anwendungen anstatt auf die Lernergebnisse. Darüber hinaus sei immersive VR bisher meist Teil experimenteller Entwicklungsarbeit, statt regelmäßigen Einsatzes in der tatsächlichen Lehre. 
Jedoch nicht nur die, durch Studien dokumentierte, Möglichkeit der Steigerung des Lernerfolgs macht den Einsatz von VR interessant für die Lehre, sondern auch die individuellen und institutionellen Vorteile, die VR-Lernumgebungen mit sich bringen. Auf individueller Ebene zeigt sich, dass das Lernen mit VR eine Steigerung der Motivation sowie des Lerninteresses hervorruft (u. a. Chavez und Bayona 2018; Parong und Mayer 2018). Darüber hinaus ermöglicht VR eine starke Fokussierung auf den Lerngegenstand, da äußere Einflüsse bei der Nutzung von HMD minimiert werden können. Außerdem ist eine individuelle Anpassung der Lernumwelt, im Sinne von Sprache, Vorlieben oder Komplexität, für oder auch durch den jeweiligen Nutzenden möglich. Auf institutioneller Ebene ermöglichen VR-Lernumgebungen, logistisch, zeitlich oder finanziell schwierige Aufgaben, Erfahrungen oder Ereignisse zu realisieren. So ist es möglich Studierende wiederholt komplexe und anspruchsvolle Aufgaben in einer sicheren Umgebung erproben zu lassen, wie beispielsweise physikalisch/chemische Versuche oder chirurgische Eingriffe, die erst ab einem bestimmten Grad an Kompetenz real durchgeführt werden können (u.a. Larsen et al. 2012). Darüber hinaus wird es Studierenden ermöglicht, Umgebungen kennenzulernen, wie beispielsweise die Tiefsee oder fremde Planeten, deren Besuch in der Realität logistisch (nahezu) unmöglich wäre (u. a. Çaliskan 2011). So können mithilfe von VR ohne großen Aufwand kognitive Fähigkeiten durch Erfahrungslernen erlangt werden, da Studierende Umgebungen, Situationen oder Abläufe direkt erleben können, die mit traditionellen Lehrmethoden nur schwierig nachgebildet werden können.

\section{Figments.nrw}

Vor dem Hintergrund der beschriebenen lehr-lernpsychologischen und mediendidaktischen Theorien sowie auf Basis der in Wirksamkeitsstudien aufgezeigten Potenziale von AR und VR-Anwendungen wird mit Figments.nrw ein offenes und modulares AR/VR-System entwickelt. Die insgesamt wachsende Bedeutung von AR/VR in Forschungs- und Lernprozessen führt zu der Forderung nach einer offenen und vereinfachten Handhabung dieser Technologien jenseits monolithischer Insellösungen, also AR/VR-Anwendungen, die lediglich einen spezifischen Anwendungsfall (z. B. eines Auftraggebers) abdecken.

\subsection{Grundidee, Vision und Zielsetzungen}

Die Vision von Figments.nrw ist es, AR/VR in der Breite der Hochschulbildung zugänglich zu machen und für unterschiedlichste Anwendungsfälle zu erschließen. Prämisse bei der Entwicklung von Figments.nrw ist, dass das Erstellen, Lehren und Teilen von didaktisch und pädagogisch aufbereiteten Inhalten in diesen angereicherten und immersiven Medien so einfach und intuitiv wie möglich sein muss: Arbeiten, die bisher Expertise in technischen Domänen erforderten (z. B. Programmierung, 3D-Design), werden abstrahiert und durch zugängliche Methoden einfacher nutzbar (siehe Abschn. 3.2.3). 
1. Ziel: Entwicklung von Figments.nrw, einer offenen und freien Software für den Einsatz von Augmented und Virtual Reality in der Hochschullehre Das Primärziel ist, die wesentlichen Funktionen zur Erstellung von AR/VR-Inhalten und deren Einsatz in der Hochschule in einer offenen und freien Lösung zur Verfügung zu stellen. Zwei ganz wesentliche Facetten hiervon, die spezifischen Benutzerrollen zugeordnet bzw. auf deren Anforderungen zugeschnitten sind, umfassen die zur Inhaltserstellung notwendigen Werkzeuge in einer Autorenumgebung und die für Lehr-/Lernaktivitäten benötigten Instrumente:

\section{- Autorenumgebung}

Die Erstellung von Bildungsinhalten für AR/VR in konventionellen Lösungen ist häufig komplex und ressourcenintensiv (wenn eigene Anwendungen in integrierten Entwicklungsumgebungen entwickelt werden), auf spezifische Anwendungsfälle beschränkt bzw. nicht erweiterbar (wenn monolithische Software verwendet wird) oder mit hohen wiederkehrenden Kosten verbunden (wenn Software-as-aService Modelle verwendet werden). Die Autorenumgebung von Figments.nrw wird vor diesem Kontext als offene und erweiterbare Komponente entwickelt, die komplexe Vorgänge der Erstellung von AR/VR Inhalten zu einfacher einsetzbaren Methoden abstrahiert.

\section{- Lehr- und Lernumgebung}

Diese Komponente wird von Lehrenden und Lernenden gleichermaßen genutzt werden, um auf Lernszenarien und andere Inhalte, die mittels der Autorenumgebung erstellt wurden, zuzugreifen. Dazu gehören nicht nur Methoden zur technischen Realisierung von AR/VR (mittels HMD, Desktop-PC oder mobilem Endgerät), sondern auch die Realisierung von Multi-User Szenarien, das Hosting und die Distribution von Lerninhalten in zentralen oder dezentralen Repositorien sowie Instrumente zur Steuerung und Durchführung von Übungen durch Lehrende.

Besteht zudem ein Bedarf an spezifischen AR/VR Lösungen in der Lehre, können durch das modulare Konzept eigene Zielsetzungen, Inhalte, Spezifikationen und Features entwickelt werden. Gleichzeitig wird durch Open-Source-Lizenzen gewährleistet, dass die so entwickelten Lösungen wiederum für Dritte bereitgestellt werden.

\section{Ziel: Demonstration der Einsatzmöglichkeiten von Figments.nrw Zur De-} monstration der Einsatzmöglichkeiten von AR/VR in der Hochschullehre und zur Evaluation von Figments.nrw werden im Projekt AR/VR.nrw exemplarische Lehrund Lernszenarien erarbeitet. Ein erstes Betätigungsfeld werden die Natur- bzw. Ingenieurwissenschaften und darin speziell die Materialwissenschaften sein. Die entstehenden Lernszenarien können in vorhandene Curricula integriert und durch die Nutzung der Autorenumgebung von Figments.nrw weiterentwickelt, verändert oder neu aufgelegt werden. Diese ist aber nicht themengebunden - Lernszenarien können für viele andere Inhalte und Fachbereiche entwickelt und realisiert werden, während die Autorenumgebung selbst durch zusätzliche Softwarekomponenten erweitert werden kann.

3. Ziel: Entwicklung und Bereitstellung von Serviceangeboten Die strukturelle Implementierung von Figments.nrw an allen Hochschulen (beginnend mit den Pro- 
jektpartnern in NRW) wird sicherstellen, dass alle Lehrenden durch ihre Servicestellen dezentrale Unterstützung bei der Entwicklung eigener AR/VR-Anwendungsfälle erhalten. Um diese übergeordneten Ziele zu erreichen, werden folgende Teilschritte bei der konzeptionellen Entwicklung und technischen Umsetzung verfolgt:

- Figments.nrw wird im Projekt AR/VR.nrw entwickelt, in iterativen Prototypen getestet, evaluiert und weiterentwickelt, wobei eine Reihe unterschiedlicher Anforderungen an die konzeptionelle und technische Umsetzung einer solchen Lösung zu beachten sind.

- Das Konzept wiederverwendbarer Figmente wird etabliert. Unter Figmenten werden in diesem Kontext aufbereitete Inhalte verstanden, die in ihrer Granularität von bearbeiteten 3D-Objekten bis zu kompletten Lernmodulen reichen können. Um den Austausch und die Wiederverwertbarkeit dieser Figmente zu ermöglichen, wird mit www.figments.nrw eine dedizierte Plattform dazu aufgebaut.

- Figmente werden in der Autorenumgebung von Figments.nrw erstellt. Diese wird eine Reihe einfach zu bedienender Werkzeuge zur Verfügung stellen, mit denen virtuelle Lernwelten gestaltet werden können. 3D-Daten können importiert, in digitalen Umgebungen positioniert sowie um interaktive Komponenten und Feedback-Mechanismen erweitert werden. Neben narrativen Inhalten werden sich offene Welten gestalten lassen, die autonom von Lernenden erkundet werden können.

- Formative Evaluationen werden die Arbeiten im Projekt AR/VR.nrw flankieren, in denen die Usability und Reliabilität entwickelter Softwarekomponenten (auf den Ebenen der Inhaltserstellung, Unterrichtsdurchführung und des Lernens) sowie die Effekte, die sich durch deren Einsatz in der Hochschulbildung generieren lassen, untersucht werden.

- Es werden Service- und Support-Angebote geschaffen, die Lehrende beim Einsatz von Figments.nrw für eigene Lehr-/Lernprojekte unterstützen. Darunter fallen z.B. Tutorials, Screencasts, eine Wissensdatenbank, Richtlinien und Tipps für die Anpassung und Weiterentwicklung der Softwarekomponenten sowie allgemeine Empfehlungen für den Einsatz von AR/VR in der Hochschulbildung.

\subsection{Anforderungen an eine AR/VR-Lösung für Hochschulen}

Im Methoden-Mix der Hochschulbildung stellen Augmented und Virtual Reality interaktive und immersive Medien dar, die eine neue und natürliche Auseinandersetzung mit digitalen Inhalten ermöglichen. Einerseits ist hier die breite Anwendbarkeit auf Ebene der Lernenden eine zu erreichende Zielgröße, andererseits müssen Werkzeuge geschaffen werden, die eine sinnvolle Integration in bereits bestehende Bildungsarrangements ermöglichen. Zielgruppen sind daher von vornherein nicht nur Lernende, sondern auch Lehrende, Fachexperten und Content-Autoren, die möglichst einfach Inhalte für das Lernen in und mit erweiterten und virtuellen Realitäten produzieren wollen. Bei der Konzeption von Figments.nrw werden folgende Anwendungsdomänen (Aspekte der technischen Entwicklung bzw. des Einsatzes der Lösung) und Anforderungsebenen zur Konkretisierung dieser Domänen herangezogen. 
Tab. 2 Anwendungsdomäne „Software-Entwicklung“

\begin{tabular}{lll}
\hline $\begin{array}{l}\text { Anwendungs- } \\
\text { domäne }\end{array}$ & Anforderungsebene & Anforderungen \\
\hline $\begin{array}{l}\text { Software- } \\
\text { Entwicklung }\end{array}$ & $\begin{array}{l}\text { Entwicklungs- } \\
\text { umgebung }\end{array}$ & $\begin{array}{l}\text { Integrierte Entwicklungsumgebung statt eigener Engine } \\
\text { Nutzen etablierter offener Branchenlösungen } \\
\end{array}$ \\
& Dokumentation & Ausführlich und transparent \\
& System-Neutralität & Unterstützung diverser Ausgabekanäle \\
& Lizenzen & Wo möglich offene und freie Lizenzen \\
\hline
\end{tabular}

Die Ebenen der Inhaltserstellung und der Visualisierung virtueller Räume stehen dabei zunächst im Fokus. So wird sichergestellt, dass die exemplarisch im Projekt generierten Inhalte und Funktionen später auch von Praxisanwendern reproduziert werden können. Mittels der Autorenumgebung von Figments.nrw werden dazu beispielhaft mehrere Lernmodule erstellt, mit denen jeweils neue Funktionen eingeführt, bestehende verbessert und Feedback aus praktischen Nutzerstudien eingepflegt werden. Es wird vermutet, dass einige grundlegende Funktionen von vornherein erfolgreich eingesetzt werden können (z.B. das einfache Greifen von Objekten in VR), diese aber basierend auf dem Feedback aus praktischen Nutzerstudien in mehreren Iterationen erweitert werden, um letztlich möglichst effektiv und effizient in der Praxis eingesetzt $\mathrm{zu}$ werden.

\subsubsection{Technische Anwendungsdomäne: Software-Entwicklung}

Figments.nrw wird in Unity programmiert. Dies hat diverse Vorteile, die im Kontext der Forschungsaktivitäten im Projekt AR/VR.nrw vor allem darin bestehen, dass Softwarekomponenten schnell entwickelt, Prototypen somit frühzeitig in praktischen Anwender-Studien evaluiert und Feedback zur iterativen Weiterentwicklung ausgewertet werden können. Es kann zudem weitestgehend systemneutral gearbeitet werden, was die Bereitstellung von Figments.nrw für unterschiedliche Ausgabemedien und -systeme begünstigt. Während zu Beginn der Softwareentwicklung der Fokus auf Windows-basierten Desktop Systemen und gekoppelter VR Hardware liegt, wird dieser je nach Bedarfslage sukzessive um Stand-Alone VR, AR, MacOS-basierte Desktop-Systeme und mobile Endgeräte erweitert. Der Quellcode wird ausführlich dokumentiert und soweit Lizenzrechte eingebundener Plug-Ins und externer Softwarekomponenten nicht verletzt werden unter Nutzung offener und freier Lizenzen veröffentlicht. Die konkreten Anforderungsebenen der Anwendungsdomäne ,Software-Entwicklung“ sind in Tab. 2 dargestellt.

\subsubsection{Technische Anwendungsdomäne: Datenmanagement}

Augmented und Virtual Reality nutzen in der Regel dreidimensionale Räume und Objekte zur Visualisierung. Es besteht daher ein erhöhter Bedarf, 3D-Daten möglichst effektiv einsetzen zu können. Die Relation zwischen Erstellungsaufwand und einem zu generierenden Mehrwert aus der Nutzung dieser Daten muss hierbei möglichst günstig ausfallen. Um auch 3D-Daten externer Quellen, die z. B. als offene 
Tab. 3 Anwendungsdomäne ,Datenmanagement“

\begin{tabular}{lll}
\hline $\begin{array}{l}\text { Anwendungs- } \\
\text { domäne }\end{array}$ & $\begin{array}{l}\text { Anforderungs- } \\
\text { ebene }\end{array}$ & Anforderungen \\
\hline $\begin{array}{l}\text { Daten- } \\
\text { management }\end{array}$ & $\begin{array}{l}\text { Figment- } \\
\text { Datenformat } \\
\text { 3D-Daten }\end{array}$ & $\begin{array}{l}\text { Offen und editierbar } \\
\text { Erweiterbare Content-Struktur und Hierarchie } \\
\end{array}$ \\
& $\begin{array}{l}\text { Unterstützung eines primären Austauschformates (.gtlf) } \\
\text { Unterstützung marktüblicher 3D- (.obj, .fbx) und CAD-Formate (.step) } \\
\text { Unterstützung von (stereoskopischen) 360 -Formaten }\end{array}$ \\
& $\begin{array}{l}\text { Konventionelle } \\
\text { Inhalte }\end{array}$ & \\
\hline
\end{tabular}

Bildungsmaterialien (OER) und/oder in Objektdatenbanken vorliegen, einbinden zu können, sollen möglichst viele der gängigen Dateiformate unterstützt werden. Ein starker Fokus liegt hierbei auf gLTF-Dateien (Graphics Language Transmission Format). gLTF-Dateien enthalten neben den für die Darstellung von 3D-Objekten notwendigen Daten (Objektgeometrie, Texturen, Materialien) zusätzliche Informationen (Lichtquellen, Kameras, Szenen-Struktur, Hierarchie, Animationen, Metadaten). Eine Vielzahl an Open Source Konvertierungstools ermöglicht die Überführung anderer Dateiformate (Collada, FBX, Obj) in das gLTF-Format. Als Container-Format eignet es sich somit besonders als Basis für Figmente, die im Verlauf des Projektes je nach Bedarfslage um weitere Formate ergänzt werden kann. Die konkreten Anforderungsebenen der Anwendungsdomäne ,Datenmanagement“ sind in Tab. 3 dargestellt.

\subsubsection{Praktische Anwendungsdomäne: Gestaltung von Lernwelten}

Da die Projektpartner ein besonderes Potenzial in der didaktischen und technischen Aufbereitung von (3D-)Daten für den Einsatz in der Hochschulbildung sehen, diese Arbeiten bisher aber spezifisches Expertenwissen in der Software-Entwicklung und der 3D-Gestaltung vorausgesetzt haben, wird Figments.nrw eine Autorenumgebung enthalten. Spezifisches technisches Prozesswissen wird hierbei in Form einfach zu bedienender Werkzeuge abstrahiert. Dazu gehören unter anderem Werkzeuge, die den Import und die Bearbeitung von 3D-Daten, die Gestaltung von VR-Umgebun-

Tab. 4 Anwendungsdomäne „Gestaltung von Lernwelten“

\begin{tabular}{lll}
\hline $\begin{array}{l}\text { Anwendungs- } \\
\text { domäne }\end{array}$ & $\begin{array}{l}\text { Anforderungs- } \\
\text { ebene }\end{array}$ & Anforderungen \\
\hline $\begin{array}{l}\text { Gestaltung } \\
\text { von Lern- }\end{array}$ & $\begin{array}{l}\text { Autorenumgebung } \\
\text { und Figment- }\end{array}$ & $\begin{array}{l}\text { Werkzeuge zur Erstellung von } \\
\text { Lelten }\end{array}$ \\
Editor & $\begin{array}{l}\text { (Persistente) Lernwelten } \\
\text { Interaktionen }\end{array}$ \\
& 3D-Editor (Rotation, Translation, Skalierung, Animation) \\
& Interaktions-Editor \\
& Feedback-Editor \\
& Simulations-Editor \\
& Rich-Text-Editor \\
& & Mehrfachverwertung vorhandener 3D-Modelle und Inhalte \\
\hline
\end{tabular}


Tab. 5 Anwendungsdomäne „Mit AR/VR lehren“

\begin{tabular}{lll}
\hline $\begin{array}{l}\text { Anwendungs- } \\
\text { domäne }\end{array}$ & $\begin{array}{l}\text { Anforderungs- } \\
\text { ebene }\end{array}$ & Anforderungen \\
\hline $\begin{array}{l}\text { Mit AR/VR } \\
\text { lehren }\end{array}$ & $\begin{array}{l}\text { Crossmedialität } \\
\text { User-Manage- } \\
\text { ment }\end{array}$ & $\begin{array}{l}\text { Unterricht über alle zur Verfügung stehenden Mediengrenzen } \\
\text { Feedbacksysteme } \\
\text { Freigabe von Funktionen } \\
\text { Aufgabenstellungen }\end{array}$ \\
& $\begin{array}{l}\text { Rechte- } \\
\text { management }\end{array}$ & $\begin{array}{l}\text { Benutzerrollen } \\
\text { Session-Ma- }\end{array}$ \\
& $\begin{array}{l}\text { Aktive Unterrichtsdurchführung } \\
\text { nagement }\end{array}$ & Mabaghementung und Begleitung \\
& &
\end{tabular}

gen, die Erstellung virtueller Instruktionen und die Erhebung von Feedback ermöglichen. Autorinnen und Autoren können diese Werkzeuge bei der Erstellung von AR/VR-Lerninhalten anwenden, um die in Kap. 2 beschriebenen Gestaltungsprinzipien der Theory of Multimedia Learning (CTML) oder der Cognitive Load Theory $(C L T)$ möglichst einfach und effektiv anzuwenden.

Die konkreten Anforderungsebenen der Anwendungsdomäne „Gestalten von Lernwelten" sind in Tab. 4 dargestellt.

\subsubsection{Praktische Anwendungsdomäne: Mit AR/VR lehren}

Insbesondere bei der Anwendungsdomäne des Lehrens mit AR/VR zeigt sich die Bedeutsamkeit der zugrundeliegenden Modelle aus Kap. 2. Denn einerseits sollten bei dem Design des Materials die Gestaltungsprinzipien der CTML und CLT berücksichtigt sowie die Implikationen aus dem Cognitive Affective Model of Immersive Learning (CAMIL) umgesetzt werden. Andererseits ist hier auch die Auseinandersetzung mit den im CAMIL enthaltenen kognitiven und affektiven Faktoren (z.B. Motivation, Interesse) von Bedeutung. Neben der Vorbereitung von Lehr- und Lernmaterialien, auf Basis dieser zentralen Planungs- und Designprinzipien, sind Funktionen und Werkzeuge notwendig, die Lehrende bei der Durchführung und Steuerung von Lernaktivitäten unterstützen. Zentrales Element ist hier die Crossmedialität von Figments.nrw. Die Partner des Projekts AR/VR.nrw haben in zahlreichen Forschungsvorhaben und Industriekooperationen die Erfahrung gemacht, dass neben der Verfügbarkeit technischer Infrastruktur (z. B. VR-Headsets) häufig für den Einsatz von AR/VR in größeren Gruppen förderliche Konzepte fehlen. Um diese Aspekte zu kompensieren müssen Lehrende besondere Unterstützung erhalten. Diese Funktionen werden sie dazu befähigen, sinnvoll mit Lernenden zu interagieren, Instruktionen im virtuellen Raum zu stellen und aktiv begleitete lineare sowie offene Lernaktivitäten durchzuführen. Die konkreten Anforderungsebenen der Anwendungsdomäne „Mit AR/VR lehren“ sind in Tab. 5 dargestellt. 
Tab. 6 Anwendungsdomäne „Mit AR/VR lernen“

\begin{tabular}{|c|c|c|}
\hline $\begin{array}{l}\text { Anwendungs- } \\
\text { domäne }\end{array}$ & $\begin{array}{l}\text { Anforderungs- } \\
\text { ebene }\end{array}$ & Anforderungen \\
\hline \multirow[t]{6}{*}{$\begin{array}{l}\text { Mit } \\
\text { AR/VR } \\
\text { lernen }\end{array}$} & Interaktion & $\begin{array}{l}\text { 3D-Zeichnungen/3D-Malen } \\
\text { Objekt-Interaktion (Greifen, Bewegen, De-/Konstruieren) } \\
\text { Navigation } \\
\text { Natural User Interfaces }\end{array}$ \\
\hline & Kollaboration & $\begin{array}{l}\text { Voice Chat } \\
\text { Symmetrische und asymmetrische Nutzer-Interaktion } \\
\text { Synchrone und asynchrone Nutzer-Kommunikation }\end{array}$ \\
\hline & Inklusion & $\begin{array}{l}\text { Alternative Methoden für Menschen mit Beeinträchtigungen } \\
\text { Eingabe } \\
\text { Navigation } \\
\text { Visuelle Reize }\end{array}$ \\
\hline & Training & $\begin{array}{l}\text { Instruktionen und Übungsaufgaben } \\
\text { Lernerfolgskontrollen }\end{array}$ \\
\hline & Feedback & $\begin{array}{l}\text { Automatisiertes Feedback zu vorab definierten Aufgabe /Aktionen } \\
\text { Feedback-Methoden für die Interaktion Lehrende-Lernende } \\
\text { Attributionales Feedback }\end{array}$ \\
\hline & $\begin{array}{l}\text { User-Profi- } \\
\text { ling }\end{array}$ & Aufzeichnung z. B. des Lernfortschritts \\
\hline
\end{tabular}

\subsubsection{Praktische Anwendungsdomäne: Mit AR/VR lernen}

Der Wandel der Rollenverständnisse im Kontext des Lehrens und Lernens mit AR/VR zeigt sich nicht nur auf Seiten der Lehrenden. Die Art des Lernens wandelt sich durch eine zunehmende Autonomie der Lernenden ebenfalls, da offen gestaltete Lernwelten Selbstbestimmung und eigene Erkundung einfordern. Ein hohes Potenzial bieten zudem spezielle Lernsettings, z. B. in Form selbstbestimmter Exploration, interaktiver Experimente oder sozialer Kollaboration.

Neben der Konzeption und Gestaltung authentischer Kontexte stellen Feedbackbasierte Unterstützungsmaßnahmen einen wichtigen Gelingensfaktor für den erfolgreichen Einsatz von AR/VR in der Bildung dar. Studien im Schulkontext zeigen, dass Feedback einer der wirksamsten Faktoren für gelingende Lernprozesse ist (Dresel und Ziegler 2006). Inhaltsspezifische und situative Hilfestellungen und Leistungsrückmeldungen sollen kognitiver Überlastung und möglichen Fehlannahmen vorbeugen. Mit der Ergänzung um attributionales Feedback, welches Erfolge durch interne Ursachen (z.B. Anstrengung) und Misserfolge durch variable, externe Ursachen (z.B. mangelndes Engagement) erklärt, werden ungünstige Ursachenzuschreibungen von Erfolg und Misserfolg im Lernprozess berücksichtigt, indem auf motivationalemotionaler Ebene die Lernleistung positiv beeinflusst wird (Reinmann 2005).

Die konkreten Anforderungsebenen der Anwendungsdomäne „Mit AR/VR lernen“ sind in Tab. 6 dargestellt. 


\section{Forschungsvorhaben und Ausblick}

Für die Entwicklung und Nutzung immersiver VR-Lernumgebungen in der Hochschullehre ist es von großer Bedeutung zentrale Ansätze der Mediendidaktik sowie der Lehr-Lernpsychologie zu berücksichtigen. Der Mehrwert, der durch die Nutzung immersiver, multimedialer Lernumgebungen bzw. Lernmaterials im Sinne der Cognitive Theory of Multimedia Learning entsteht und durch verschiedene Studien belegt werden konnte (für einen Überblick siehe u. a. Hamilton et al. 2021 und Radianti et al. 2020), ist schlüssig und überzeugend. Diese Nutzung sollte jedoch stets vor dem Hintergrund der Cognitive Load Theory und entsprechenden Gestaltungsprinzipien hinterfragt und begleitet von einer gezielten didaktischen Planung, z. B. orientiert am Cognitive Affective Model of Immersive Learning, umgesetzt werden. So kann und sollte ein sinnvolles technisch-didaktisches Konzept entwickelt werden, dass das Potenzial der Lernumgebung und des Lernmaterials umfänglich ausschöpft, kognitive sowie motivationale und affektive Prozesse positiv beeinflusst und damit das (Erfahrungs-)Lernen fördert.

Mit Figments.nrw werden zukünftig die bestehenden Hürden einer stärkeren Integration der Technologien AR/VR in Lehre und Forschung der NRW-Hochschulen minimiert sowie die Kollaboration beteiligter Stakeholder und Akteure im Bereich AR/VR verbessert. Die nutzerfreundliche Handhabung der Software (sowohl der Autorenumgebung als auch der Lehr-/Lernumgebung) sowie die modulare Softwareentwicklung nach freien und offenen Standards, wird die breite Anwendung von AR/VR in der Hochschulbildung begünstigt. Exemplarisch erarbeitete Lernszenarien dienen nicht nur als Grundlage zur Darstellung des Funktionsumfangs der Software, sondern zeigen potenziellen Anwendern Möglichkeiten zur sinnvollen und sinnstiftenden Integration von AR/VR in eigenen Lernarrangements auf. Zusätzliche Services zur Unterstützung der Anwenderinnen und Anwender aus der Praxis werden im Projekt AR/VR.nrw aufgebaut und angeboten, um die Nutzung bestehender und Entwicklung eigener AR/VR Lehr- und Lerninhalte zu erleichtern.

Die Softwareentwicklung von Figments.nrw wird um verschiedene Forschungsvorhaben, vor allem zur Evaluation der entwickelten Lösung, ergänzt. Dabei sollen verschiedene Bereiche, Zielgruppen und Fragestellungen abgedeckt werden. Dazu werden Evaluationsstudien zu den Themenbereichen Usability, Akzeptanz und Wirksamkeit, sowohl mit Lehrenden als auch mit Studierenden durchgeführt. Im Rahmen von Usability-Studien beurteilen Lehrende und Studierende die Nutzerfreundlichkeit des Systems, indem sie dieses zum einen erkunden können und zum anderen mögliche Aufgabenstellungen erfüllen sollen. Lehrende werden darüber hinaus gebeten, mithilfe der Autorenwerkzeuge, eigene Inhalte und Umgebungen zu kreieren. Im Rahmen von Akzeptanzstudien machen Lehrende und Studierende Angaben zur potenziellen Nutzung und Einstellung gegenüber Figments.nrw, um so eine mögliche Annahmeentscheidung der Innovation zu identifizieren. Im Rahmen von Wirksamkeitsstudien werden mithilfe eines experimentellen Gruppen-Designs Studierende begleitet und befragt, welche im Rahmen einer Lehrveranstaltung mit Figments.nrw konfrontiert werden. Gemessen werden in diesem Rahmen sowohl affektive, motivationale und kognitive Prozesse/Outcomes als auch Lernergebnisse 
der Studierenden. Die Ergebnisse der Evaluationen fließen in die Modifikation und Weiterentwicklung des Systems ein.

Die ersten praktischen Erprobungen von Figments.nrw sind für das Wintersemester 2021/2022 geplant, über die das Projektteam auf www.figments.nrw rechtzeitig berichten wird.

Danksagung Das Forschungsvorhaben AR/VR.nrw wird von einem Verbund der Hochschule Rhein-Sieg, der RWTH Aachen, der Bergischen Universität Wuppertal und der Hochschule Hamm-Lippstadt begleitet. Die Autoren bedanken sich beim Ministerium für Kultur und Wissenschaft NRW für finanzielle Förderung im Rahmen des Förderprogramms Digitale Hochschule NRW (DH.NRW) und allen Projektbeteiligten für die Unterstützung des Vorhabens.

Funding Open Access funding enabled and organized by Projekt DEAL.

Open Access Dieser Artikel wird unter der Creative Commons Namensnennung 4.0 International Lizenz veröffentlicht, welche die Nutzung, Vervielfältigung, Bearbeitung, Verbreitung und Wiedergabe in jeglichem Medium und Format erlaubt, sofern Sie den/die ursprünglichen Autor(en) und die Quelle ordnungsgemäß nennen, einen Link zur Creative Commons Lizenz beifügen und angeben, ob Änderungen vorgenommen wurden.

Die in diesem Artikel enthaltenen Bilder und sonstiges Drittmaterial unterliegen ebenfalls der genannten Creative Commons Lizenz, sofern sich aus der Abbildungslegende nichts anderes ergibt. Sofern das betreffende Material nicht unter der genannten Creative Commons Lizenz steht und die betreffende Handlung nicht nach gesetzlichen Vorschriften erlaubt ist, ist für die oben aufgeführten Weiterverwendungen des Materials die Einwilligung des jeweiligen Rechteinhabers einzuholen.

Weitere Details zur Lizenz entnehmen Sie bitte der Lizenzinformation auf http://creativecommons.org/ licenses/by/4.0/deed.de.

\section{Literatur}

Azuma R, Baillot Y, Behringer R, Feiner S, Julier S, MacIntyre B (2001) Recent advances in augmented reality. IEEE Comput Grap Appl 21(6):34-47. https://doi.org/10.1109/38.963459

Baddeley A (2012) Working memory: theories, models, and controversies. Annu Rev Psychol 63:1-29. https://doi.org/10.1146/annurev-psych-120710-100422

Blade RA, Padgett ML (2015) Virtual environments standards and terminology. In: Hale KS, Stanney KM (Hrsg) Handbook of virtual environments: Design, implementation, and applications. CRC Press, S 23-35

Buttussi F, Chittaro L (2018) Effects of different types of virtual reality display on presence and learning in a safety training scenario. IEEE Trans Visual Comput Graphics 24(2):1063-1076. https://doi.org/ 10.1109/TVCG.2017.2653117

Çaliskan O (2011) Virtual field trips in education of earth and environmental sciences. Proc Soc Behav Sci 15:3239-3243. https://doi.org/10.1016/j.sbspro.2011.04.278

Chavez B, Bayona S (2018) Virtual reality in the learning process. In: Rocha A, Adeli H, Reis LP, Constanzo S (Hrsg) Advances in intelligent systems and computing. Trends and advances in information systems and technologies. Springer, Berlin Heidelberg, S 1345-1356 https://doi.org/10.1007/978-3319-77712-2_129

Clark JM, Paivio A (1991) Dual coding theory and education. Educ Psychol Rev 3(3):149-210. https://doi. org/10.1007/BF01320076

Dörner R, Jung B, Grimm P, Broll W, Göbel M (2013) Einleitung. In: Dörner R, Broll W, Grimm P, Jung B (Hrsg) Virtual und Augmented Reality (VR/AR): Grundlagen und Methoden der Virtuellen und Augmentierten Realität. Springer Vieweg, , S 1-32 https://doi.org/10.1007/978-3-642-28903-3

Dresel M, Ziegler A (2006) Langfristige Förderung von Fähigkeitsselbstkonzept und impliziter Fähigkeitstheorie durch computerbasiertes attributionales Feedback [Long-term enhancement of academic self-concept and implicit ability theory through computer-based attributional feedback]. Z Pädagog Psychol 20(1):49-63. https://doi.org/10.1024/1010-0652.20.12.49 
Goertz L, Fehling CD, Hagenhofer T (2021) Didaktische Konzepte identifizieren - Community of Practice zum Lernen mit AR und VR. www.social-augmented-learning.de/wp-content/downloads/210225Coplar-Leitfaden_final.pdf.. Zugegriffen: 14. Juli 2021

Hamilton D, McKechnie J, Edgerton E, Wilson C (2021) Immersive virtual reality as a pedagogical tool in education: a systematic literature review of quantitative learning outcomes and experimental design. J Comput Educ 8(1):1-32. https://doi.org/10.1007/s40692-020-00169-2

Hellriegel J, Čubela D (2018) Das Potenzial von Virtual Reality für den schulischen Unterricht: Eine konstruktivistische Sicht. MedienPädagogik. https://doi.org/10.21240/mpaed/00/2018.12.11.X

Hochberg J, Vogel C, Bastiaens T (2017) Gestaltung und Erforschung eines Mixed-Reality-Lernsystems. MedienPädagogik 28:40-46. https://doi.org/10.21240/mpaed/28/2017.03.21.X

Johnston APR, Rae J, Ariotti N et al (2018) Journey to the centre of the cell: virtual reality immersion into scientific data. Traffic 19(2):105-110. https://doi.org/10.1111/tra.12538

Kerres M (2018) Mediendidaktik: Konzeption und Entwicklung digitaler Lernangebote. De Gruyter, https://doi.org/10.1515/9783110456837

Köhler T, Münster S, Schlenker L (2013) Didaktik virtueller Realität: Ansätze für eine zielgruppengerechte Gestaltung im Kontext akademischer Bildung. In: Reinmann G, Ebner M, Schön S (Hrsg) Hochschuldidaktik im Zeichen von Heterogenität und Vielfalt: Doppelfestschrift für Peter Baumgartner und Rolf Schulmeister. Books on Demand, , S 99-112

Larsen CR, Oestergaard J, Ottesen BS, Soerensen JL (2012) The efficacy of virtual reality simulation training in laparoscopy: a systematic review of randomized trials. Acta Obstet Gynecol Scand 91(9):1015-1028. https://doi.org/10.1111/j.1600-0412.2012.01482.x

Lee H, Plass JL, Homer BD (2006) Optimizing cognitive load for learning from computer-based science simulations. J Educ Psychol 98(4):902-913. https://doi.org/10.1037/0022-0663.98.4.902

Makransky G, Petersen GB (2021) The cognitive affective model of immersive learning (CAMIL): a theoretical research-based model of learning in immersive virtual reality. Educ Psychol Rev. https://doi. org/10.1007/s10648-020-09586-2

Mayer RE (2014a) The cambridge handbook of multimedia learning. Cambridge University Press, https:// doi.org/10.1017/CBO9781139547369

Mayer RE (2014b) Cognitive theory of multimedia learning. In: Mayer RE (Hrsg) The cambridge handbook of multimedia learning. Cambridge University Press, , S 43-71 https://doi.org/10.1017/ CBO9781139547369.005

Mayer RE, Fiorella L (2014) Principles for reducing extraneous processing in multimedia learning: coherence, signaling, redundancy, spatial contiguity, and temporal contiguity principles. In: Mayer RE (Hrsg) The Cambridge handbook of multimedia learning. Cambridge University Press, , S 279-315

Moreno R, Mayer RE (2002) Learning science in virtual reality multimedia environments: role of methods and media. J Educ Psychol 94(3):598-610. https://doi.org/10.1037/0022-0663.94.3.598

Niedermeier S, Müller-Kreiner C (2019) VR/AR in der Lehre!? Eine Übersichtsstudie zu Zukunftsvisionen des digitalen Lernens aus der Sicht von Studierenden. https://www.pedocs.de/volltexte/2019/18048. Zugegriffen: 16. Juli 2021

Paas F, Sweller J (2014) Implications of cognitive load theory for multimedia learning. In: Mayer RE (Hrsg) The Cambridge handbook of multimedia learning. Cambridge University Press, Cambridge, S $27-42$

Paas F, Renkl A, Sweller J (2003) Cognitive load theory and instructional design: recent developments. HEDP 38(1):1-4. https://doi.org/10.1207/S15326985EP3801_1

Parong J, Mayer RE (2018) Learning science in immersive virtual reality. J Educ Psychol 110(6):785-797. https://doi.org/10.1037/edu0000241

Radianti J, Majchrzak TA, Fromm J, Wohlgenannt I (2020) A systematic review of immersive virtual reality applications for higher education: design elements, lessons learned, and research agenda. Comput Educ 147:103778. https://doi.org/10.1016/j.compedu.2019.103778

Reinmann G (2005) Innovation ohne Forschung? Ein Plädoyer für den Design-Based Research-Ansatz in der Lehr-Lernforschung. Unterrichtswissenschaft 33(1):52-69

Sweller J (2010) Cognitive load theory: recent theoretical advances. In: Plass JL, Moreno R, Brunken R (Hrsg) Cognitive load theory. Cambridge University Press, Cambridge, S 29-47 https://doi.org/10. 1017/CBO9780511844744.004

Sweller J, Chandler P (1994) Why some material is difficult to learn. Cogn Instr 12(3):185-233. https:// doi.org/10.1207/s1532690xci1203_1 
Wittrock MC (1989) Generative processes of comprehension. Educ Psychol 24(4):345-376. https://doi. org/10.1207/s15326985ep2404_2

Zender R, Weise M, von der Heyde M, Söbke H (2018) Lehren und Lernen mit VR und AR - Was wird erwartet? Was funktioniert? In: Schiffner D (Hrsg) Die 16. E-Learning Fachtagung Informatik (DeLFi), Frankfurt, Deutschland. http://ceur-ws.org/Vol-2250/WS_VRAR_paper5.pdf. Zugegriffen: 16. Juli 2021 